\title{
IMPROVEMENTS IN CURING COUPLED BUNCH INSTABILITIES AT ELETTRA BY MODE SHIFTING AFTER THE INSTALLATION OF THE ADJUSTABLE HIGHER ORDER MODE FREQUENCY SHIFTER (HOMFS).
}

\author{
M.Svandrlik, A.Fabris, C.Pasotti, Sincrotrone Trieste, S.S. 14 km 163.5, Basovizza, Trieste, Italy
}

\section{Abstract}

Mode shifting is the technique used at the third generation synchrotron light source ELETTRA to cure Coupled Bunch Instabilities (CBI). Temperature tuning of the RF cavities, based on an analytical prediction algorithm, allows longitudinal stable operating conditions to be obtained. However, once longitudinal stability has been achieved transverse instabilities can be observed, particularly during the energy ramp from 1.0 to $2.0 \mathrm{GeV}$. There has therefore been the need to extend the flexibility of the mode shifting technique. This has been achieved by the installation of adjustable HOMFS on the storage ring RF cavities. The HOMFS is effective on those longitudinal and transverse cavity Higher Order Modes which are unstable over a wide temperature range, by shifting them out of the operating temperature interval of the cavity. The installation of the HOMFS has improved the reliability and reproducibility of machine operation, also in view of the beam current upgrade.

\section{CURING CBI BY HOM SHIFTING}

The technique of curing CBI by shifting the frequency of the Higher Order Modes (HOM) of the RF cavities can be an interesting solution, particularly for high energy and compact storage rings. At ELETTRA the distribution of the Coupled Bunch Modes (CBM) and their growth rates have been calculated as a function of cavity temperature [1], [2]. This allows a quite flexible operation of the storage ring, with the possibility of a lifetime increase by controlled use of CBI which lengthen the bunch [3]. Characterisation of the excitation of Low Frequency Oscillations for different levels of CBI has also been possible [4]. Secondary effects on the RF cavities like cross-talk between cavities, cavity temperature oscillation and spurious vacuum interlocks could be observed and understood [5].

\subsection{Mode shifting by temperature tuning}

Cavity temperature intervals where the growth rates of all CBM are below the radiation damping level, which is a conservative condition to achieve stability, are more easily available if :

- $\quad$ the radiation damping time is short;

- the width of the temperature interval where a cavity

HOM doesn't drive any unstable CBM is large;

- $\quad$ the number of HOMs one has to deal with is small;

- the temperature tuning range of the cavity is wide

The first two conditions are more easily met by small, high energy rings. The RF cavities should not be provided with any HOM damping system, so that the HOM resonances are still sharp, high $\mathrm{Q}$ resonances. The experience at ELETTRA shows that the number of modes one has to deal with does not exceed about ten for the longitudinal case and ten for the transverse case (considering the double polarisation for the dipole modes). A cavity temperature tuning range of about $30^{\circ} \mathrm{C}$, as used in ELETTRA, is usually large enough to provide stable temperature windows. However, even a small bandwidth HOM can be unstable over a wide temperature range, if its temperature coefficient $\tau$ is low. Then the temperature tuning method can fail. This was the case for the first longitudinal mode, L1, of the ELETTRA cavity named S3. The solution was to shift L1 out from the temperature range, by installing a fixed HOMFS [2].

\subsection{Need for a further degree of freedom}

Following the installation of the fixed HOMFS, longitudinal stable intervals could be found. Transverse effects then became evident, particularly during the energy ramping from 1.0 to $2.0 \mathrm{GeV}$, when the beam begins to stabilize longitudinally, while it is still quite sensitive in the transverse plane, due to the lower natural damping available. The origin of these effects could be identified with the horizontal CBM 318, excited by the third dipole mode in cavity S9 [5]. At the same time there were still problems with cavity S3, where the largest stable longitudinal interval showed again a transverse CBM excited, as can be seen in fig. 1 and 2 . The other stable interval, towards $70{ }^{\circ} \mathrm{C}$, resulted smaller than expected in [2], due to difficulties in measuring the frequency of HOM L5, whose actual position in temperature was a couple of degrees lower than expected.

This shows the need for an additional degree of freedom to effectively cure CBI by mode shifting in ELETTRA. An adjustable HOMFS could give more flexibility in getting stable longitudinal working points, not affected by transverse effects, also in view of a beam current increase.

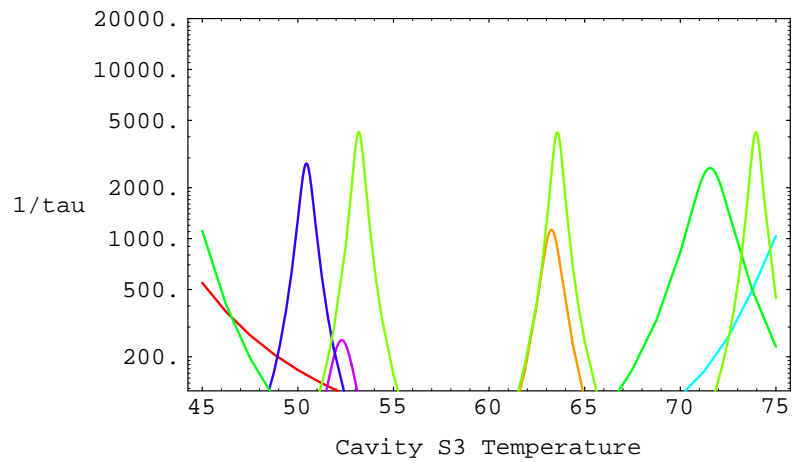

Fig. 1: Longitudinal CBMs, fixed HOMFS on S3, $2 \mathrm{GeV}, 250 \mathrm{~mA}$ 


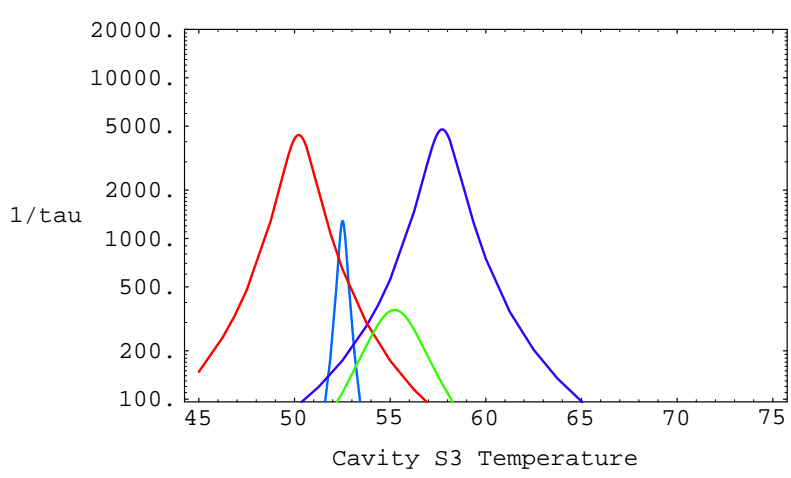

Fig. 2: Horizontal CBMs, fixed HOMFS on S3, $2 \mathrm{GeV}, 250 \mathrm{~mA}$

\section{THE ADJUSTABLE HOMFS}

\subsection{Longitudinal modes}

The adjustable HOMFS is installed on one of the three equatorial ports available on the ELETTRA cavity. It is a copper cylinder of $81 \mathrm{~mm}$ diameter, driven by a stepping motor. The range goes from 0 to $34 \mathrm{~mm}$; at its deepest position, $+34 \mathrm{~mm}$, it enters $10 \mathrm{~mm}$ inside the cavity. The frequency shifts introduced by the HOMFS itself, $\Delta \mathrm{f}_{\mathrm{HFS}}$, depend on the intensity of the field of the specific HOM in the equatorial region of the cavity. For many of the considered HOMs, this field is quite low in that region, as it can be seen from a simulation with URMEL. The second column of table 1 lists the maximum obtained frequency shifts for the relevant longitudinal HOMs, as measured on cavity S3. Modes with stronger field in the HOMFS region like L2, L3, L5 and L6 show a maximum shift of more than $1000 \mathrm{kHz}$. The shift on mode L1, one of the modes for which the HOMFS is thought, is equal to $-428 \mathrm{kHz}$.

Table 1: Adjustable HOMFS effect on longitudinal HOM

\begin{tabular}{||c|r|r|r||r|r||}
\hline \hline HOM & $\begin{array}{c}\Delta \mathrm{fHFS} \\
\mathrm{kHz}\end{array}$ & $\varphi_{\kappa}$ & $\begin{array}{c}\Delta \mathrm{f} \text { tot } \\
\mathrm{kHz}\end{array}$ & $\begin{array}{r}\tau_{\kappa} \\
\mathrm{kHz} /{ }^{\circ} \mathrm{C}\end{array}$ & \multicolumn{1}{|c||}{$\begin{array}{r}\Delta \mathrm{T} \\
{ }^{\circ} \mathrm{C}\end{array}$} \\
\hline L0 & 823 & 1.00 & 0 & & \\
L1 & -428 & 0.63 & -946 & -11.5 & 82.3 \\
L2 & 3459 & -0.31 & 3714 & -19.3 & -192.4 \\
L3 & -1567 & -2.16 & 211 & -43.1 & -4.9 \\
L4 & 683 & -0.31 & 938 & -28.2 & -33.3 \\
L5 & 4529 & -2.33 & 6447 & -41.6 & -155.0 \\
L6 & -1288 & -0.14 & -1173 & -33.4 & 35.1 \\
L7 & -140 & -2.30 & 1753 & -51.2 & -34.2 \\
L9 & 0 & -9.30 & 7654 & -111.4 & -68.7 \\
\hline
\end{tabular}

There is however a second contribution to the frequency shift generated on the HOMs. This comes from the external tuning cage, acting on the axial length of the cavity to recover the frequency shift $\Delta \mathrm{f}_{\mathrm{HFSL}_{0}}$ caused by the HOMFS on the fundamental mode, L0. The total frequency shift on the HOMs, $\Delta \mathrm{f}_{\text {tot }}$, is therefore given by

$$
\Delta \mathrm{f}_{\text {tot }}=\Delta \mathrm{f}_{\mathrm{HFS}}-\varphi_{\mathrm{k}} \Delta \mathrm{f}_{\mathrm{HFSL}_{0}} .
$$

$\varphi_{\mathrm{k}}$ is the HOM frequency shift corresponding to a unitary frequency change of L0 by the axial tuning cage, as defined and listed in [5] for all HOMs. The third column in table 1 shows the total frequency shifts for the longitudinal modes when the HOMFS is at the $+34 \mathrm{~mm}$ position. The mapping of the total frequency shifts versus the most significant range of the HOMFS is shown in fig. 3 .

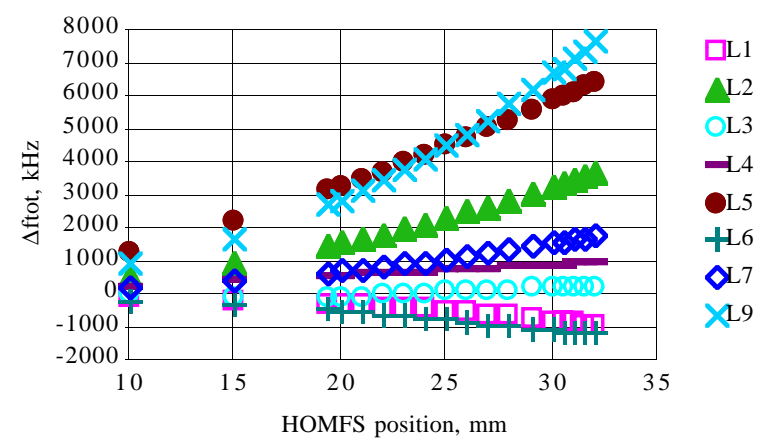

Fig. 3: Total frequency shift

If $\Delta \mathrm{f}_{\mathrm{HFS}}$ and $\varphi_{\mathrm{k}}$ are opposite in sign, the corresponding mode shows an increased frequency shift. This is particularly important for mode L1, for which the frequency shift is now more than doubled. The shift on L9 becomes quite impressive due to the large value of $\varphi_{\mathrm{k}}$. On the contrary the shift on L3 is now reduced, since the effect of the tuning cage is opposite to that of the HOMFS. Finally the effect which is of interest for the mode shifting technique is the equivalent shift in temperature for any CBM excited by one of the considered HOM. It can be calculated as

$$
\Delta \mathrm{T}=\frac{\Delta \mathrm{f}_{\text {tot }}}{\tau_{\mathrm{k}}}
$$

Thanks to the lowest temperature coefficient $\tau_{\mathrm{k}}$, the effect on HOM L1 results fairly large in temperature. Any position beyond $20 \mathrm{~mm}$ is sufficient to shift out the mode L1 from the temperature range of the cavity.

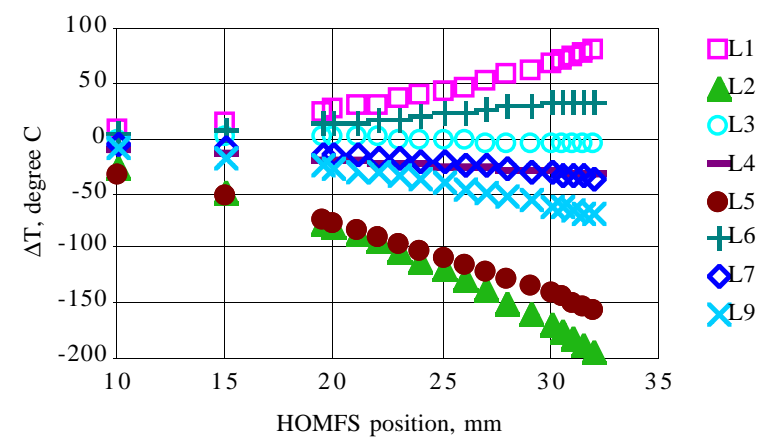

Fig. 4: Equivalent shift in temperature for the CBM excited by the longitudinal modes listed on the right.

Fig. 4 shows that only mode L3 is little affected by the HOMFS. This is not a problem since it is only unstable in a small temperature interval. Further this is the mode 
which is used to introduce a reduced longitudinal excitation to increase the lifetime in the relaxed operation of the machine.

\subsection{Transverse modes}

For the transverse modes the installation of the adjustable HOMFS on cavity $\mathrm{S} 3$ results in the temperature shifts shown in fig. 5 for the relevant modes.

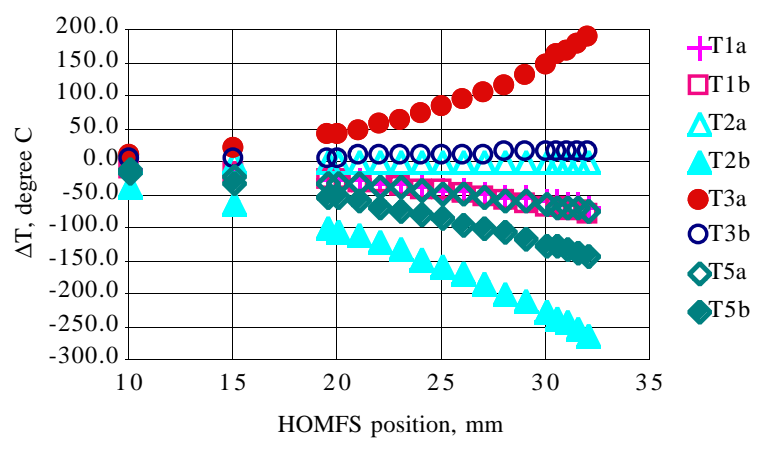

Fig. 5: Temperature shifts for the transverse CBMs

The transverse mode $\mathrm{T} 2 \mathrm{a}$ results to be the only one which is almost untouched by the HOMFS, having almost zero field in the equatorial region and also $\varphi_{\mathrm{T} 2 \mathrm{a}}=0.0$. Also $\mathrm{T} 3 \mathrm{~b}$ is little affected by the HOMFS. Both are strong impedance modes, but due to the low temperature coefficient $\left(-13.5 \mathrm{kHz} /{ }^{\circ} \mathrm{C}\right.$ for $\mathrm{T} 2 \mathrm{a},-16.1 \mathrm{kHz} /{ }^{\circ} \mathrm{C}$ for $\left.\mathrm{T} 3 \mathrm{~b}\right)$ the temperature interval between the interactions of those HOMs with two adjacent CBMs is large $\left(60-70{ }^{\circ} \mathrm{C}\right)$.

\section{IMPROVEMENTS ON THE MACHINE}

The installation of the adjustable HOMFS on cavity S9 in September 96 eliminates the problems during energy ramping, as described in [6]. Furthermore, for users' operation, the controlled excitation of the longitudinal mode L3 can be done in a more selective way, without need of adjustments for different beam current intensities.

In February 97 the second adjustable HOMFS has been installed on cavity S3 to replace the fixed version. The improvement is clearly shown in fig. 6 and fig. 7, where the CBM excitation for the longitudinal and transverse horizontal case is drawn. They are computed for $2.0 \mathrm{GeV}$, $250 \mathrm{~mA}$, with the present position of the HOMFS, +27 $\mathrm{mm}$. Now a large stable window exists for longitudinal $\mathrm{CBMs}$ in the $70^{\circ} \mathrm{C}$ temperature region of cavity S3 (fig. 6 ). At the same time fig. 7 shows that this temperature region is free of interactions with the horizontal spectrum $\left(\mathrm{Q}_{\mathrm{X}}=14.300\right)$.

\section{CONCLUSIONS}

A significant improvement in the mode shifting technique to cure CBIs has been obtained after installing two adjustable HOMFS. Transverse effects during the energy ramping are no more observed. Larger stable intervals in the longitudinal plane allow a more flexible and selective operation of the machine. Further installation of adjustable HOMFS on the other two cavities is foreseen in the near future, also in view of a beam current increase.

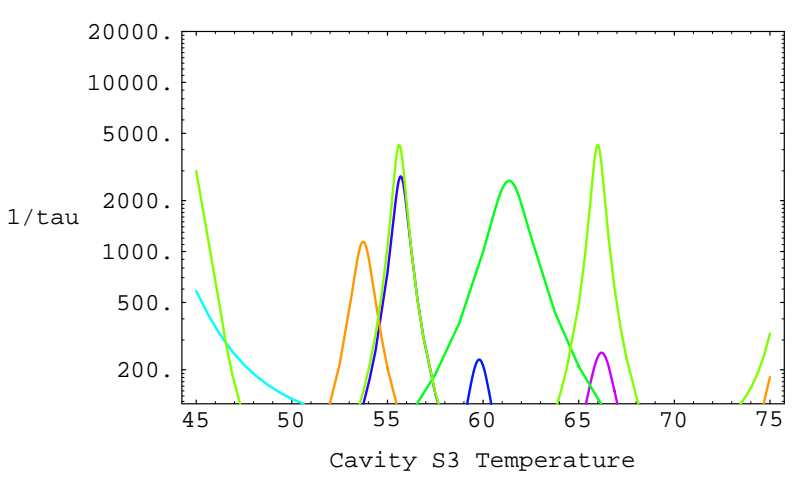

Fig. 6: Longitudinal CBMs, HOMFS + $27 \mathrm{~mm}$.

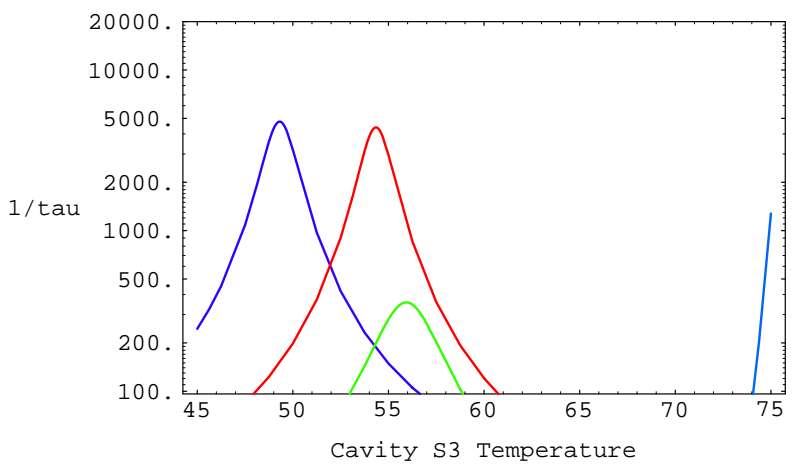

Fig. 7: Transverse Horizontal CBMs, HOMFS +27 mm.

\section{REFERENCES}

[1] M.Svandrlik et al., "The cure of Multibunch Instabilities in ELETTRA", in Proceedings of the 1995 Particle Accelerator Conference, Dallas, USA, May 1995, pp. 2762-2764.

[2] M.Svandrlik et al., "Improved Methods of Measuring and Curing Multibunch Instabilities in ELETTRA", in Proceedings of the 1996 European Particle Accelerator Conference, Sitges (Barcelona), Spain, June 1996, pp. 1144-1146.

[3] C.J.Bocchetta et al., "Operational Experience with ELETTRA", in Proceedings of the 1996 European Particle Accelerator Conference, Sitges (Barcelona), Spain, June 1996, pp. 76-80.

[4] A.Wrulich et al., "Observations of CBI in ELETTRA", in Proceedings of the 1996 European Particle Accelerator Conference, Sitges (Barcelona), Spain, June 1996, pp. 1108-1110.

[5] M.Svandrlik et al., "Simulations and Measurements of Higher Order Modes of the ELETTRA RF Cavities in view of Coupled Bunch Instability Compensation by Temperature Variation", in Proceedings of the 1996 European Particle Accelerator Conference, Sitges (Barcelona), Spain, June 1996, pp. 2053-2055.

[6] C.J.Bocchetta et al., "ELETTRA Performance and Upgrade", this conference. 